\title{
Augmented inhibition of angiogenesis by combination of HER2 antibody chA21 and trastuzumab in human ovarian carcinoma xenograft
}

Anli Zhang ${ }^{1 \dagger}$, Guodong Shen ${ }^{2,3+}$, Ting Zhao ${ }^{4}$, Guihong Zhang ${ }^{1}$, Jing Liu ${ }^{5}$, Lihua Song ${ }^{2,4}$, Wei Wei ${ }^{2}$, Ling Bing ${ }^{3}$, Zhengsheng $\mathrm{Wu}^{1}$, Qiang $\mathrm{Wu}^{\mathrm{1}^{*}}$

\begin{abstract}
Background: chA21 is a novel tumor-inhibitory antibody which recognized subdomain I of HER2 extracellular domain with an epitope distinct from other HER2 antibodies. Previously, we demonstrated that chA21 inhibits human ovarian carcinoma cell line SKOV-3 growth in vitro and in vivo study. In this study, we further investigated the anti-angiogenic efficacy combination of chA21 with trastuzumab in SKOV-3 xenograft model.

Methods: Nude mice were s.c. challenged with SKOV-3 cells and received treatment of chA21 alone, trastuzumab alone or both antibodies together twice a week for 21 days. Tumor volume and microvessel density (MVD) were evaluated. The effect of chA21 plus trastuzumab treament on vascular endothelial growth factor (VEGF) secretion, endothelial cells proliferation and migration, and the status of HER2 downstream pathway AKT/phosphorylated AKT (pAKT) were evaluated in vitro.

Results: In vivo study combination of chA21 with trastuzumab resulted in reduce tumor growth and angiogenesis than each monotherapy. In vitro study, the combination of chA21 with trastuzumab inhibits VEGF secretion, endothelial cells proliferation and migration. Furthermore, the combination treatment inhibits PAKT expression.

Conclusion: Our findings suggested that the combination of chA21 with trastuzumab can cause augmented inhibition of angiogenesis in SKOV-3 xenograft model. Inhibition of agniogenesis may through suppression of AKT pathway. The therapeutic benefits of combination chA21 with trastuzumab warrant further study in an attempt to make the translation into the clinic.
\end{abstract}

\section{Introduction}

Epithelial ovarian carcinoma is the most lethal gynecologic malignancy and resulting in high mortality rates among women patients [1]. Despite the advances in surgery, chemotherapy and radiotherapy, the average time of clinical remission is 2.5 years and approximately $20 \%$ of patients never achieve remission [2]. Thus it underscores the need for new therapeutic strategies that can be translated to the clinical treatment.

\footnotetext{
* Correspondence: aydjohn@yahoo.com

+ Contributed equally

'Department of Pathology, Anhui Medical University, Meishan Road, Hefei, China

Full list of author information is available at the end of the article
}

HER2, also named ErbB2/p185 her2/neu, is a key member of the epidermal growth factor receptor (EGFR) family. Overexpression of HER2 is associated with tumor metastasis and poor prognosis [3]. HER2 overexpression has been reported to in $15 \%$ to $30 \%$ of ovarian carcinoma patients $[4,5]$. HER2-targeted therapy with monoclonal antibodies (mAbs) is a promising strategy for the ovarian carcinoma, although trastuzumab (trademark: herceptin, Genetech, Roche) has not got such great success in ovarian carcinoma as in breast or gastric cancer [6,7].

Previously we have developed a new HER2 mAb A21. This new antibody is a single-chain chimeric derivatives of chA21, which recognizes a conformational epitope
C Biomed Central

C 2010 Zhang et al; licensee BioMed Central Ltd. This is an Open Access article distributed under the terms of the Creative Commons Attribution License (http://creativecommons.org/licenses/by/2.0), which permits unrestricted use, distribution, and reproduction in any medium, provided the original work is properly cited. 
distinct from trastuzumab and other HER2 therapeutic antibodies, thus it may represents a novel target site for HER2 therapeutics [8-11].

It is well accepted that angiogenesis plays a key role in tumor growth and metastasis. Research has shown that HER2 signaling is invovled in angiogenesis $[12,13]$. HER2 antibody trastuzumab have been shown to inhibit angiogenesis in HER2-overexpressing tumor cells [14]. The HER2 phosphorylates downstream substrates and activates a variety of signaling cascades, including the phosphatidylinositol-3 kinase (PI3K)/serine/threoninespecific protein kinase (AKT), and it regulates various cell functions especially in tumor growth, and angiogenesis [15].

In a previous study, we had found chA21 monotherapy could inhibit human ovarian carcinoma cell line SKOV-3 growth in vitro and in vivo [16]. In this study, we further investigated if more effective inhibition of angiogenesis is one of the underlying causes of the better therapeutic efficacy of the chA21 with trastuzumab combination in SKOV-3 xenograft model.

\section{Materials and methods}

\section{Humanized monoclonal antibodies and cell lines}

HER2 antibody chA21 was prepared as described in previous study [8]. Trastuzumab was purchased from Roche company (Shanghai, China).

Human ovarian carcinoma cell line SKOV-3 and human umbilical vein endothelial cells (HUVECs) were obtained from the American Type Culture Collection. SKOV-3 cells were cultured in RPMI 1640 (Gibco, USA) supplemented with $10 \%$ fetal bovine serum (Gibco, USA). HUVECs were maintained in F-12 nutrient mixture (Invitrogen, USA) enriched with 10\% newborn calf serum (Invitrogen, USA).

\section{Mice xenograft model}

Female BALB/c nude mice at 6-8 weeks of age were purchased from Nanjing Laboratory Animal Center of China. The experimental animal study protocols were approved by the Committee for Ethics in Animal Experimentation in University of Science and Technology of China. For tumor xenograft model, mice were subcutaneously injected with $5 \times 10^{6}$ SKOV-3 cells into the left flank. After inoculation, animals were weighed and tumor sizes were measured twice a week with calipers. Tumor volumes were calculated by the formula: $(\text { smaller diameter })^{2} \times$ larger diameter $\times 0.5$. When tumor volume reached about $70 \mathrm{~mm}^{3}$, the mice bearing xenografts were randomly assigned into four groups ( $\mathrm{n}$ = 8): normal saline control, chA21 alone $(30 \mathrm{mg} / \mathrm{kg})$, trastuzumab alone $(20 \mathrm{mg} / \mathrm{kg})$, and chA21 plus trastuzu$\mathrm{mab}(30 \mathrm{mg} / \mathrm{kg}+20 \mathrm{mg} / \mathrm{kg})$. Drug were delivered twice a week via caudal vein. All animals were killed after treatment for 21 days. The tumors were removed, weighed and fixed in $10 \%$ neutral buffered formalin for pathological study. The tumor inhibition ratio (TIR) was calculated as previous study: (1-experimental tumor mean weight/control tumor mean weight) $\times 100 \%$ [17]

\section{Immunohistochemistry examination}

The sections of paraffin-embedded tissue from SKOV-3 nude mice xenografts were dewaxed and rehydrated. Immunohistochemistry procedure was performed using DAKO Envision Plus kit (DAKO) according to the manufacturer's instructions. After antigen retrieval with autoclaving in citric acid, and inactivating endogenous peroxidase with $3 \% \mathrm{H}_{2} \mathrm{O}_{2}$, the slides were incubated with the rabbit anti-mouse antibody CD34 (1: 200, Bioss, China) or the rabbit anti-human antibody VEGF (working solution, ZhongShan, China) overnight at $4^{\circ} \mathrm{C}$. Second antibody conjugated with peroxidase labeled polymer was applied for $30 \mathrm{~min}$ at room temperature. The sections were developed in 3,3-diaminobenzidine and counterstained with hematoxylin. As a negative control, sections were stained normal human serum instead of the primary antibody. The mean optical density (MOD) was quantitatively analyzed using Image-pro Plus 5.02 (Media Cybernetics Inc, USA) for VEGF expression. MVD was determined by counting the number of microvessels (marked by CD34 staining) per high-power field $(200 \times)$ in the sections as previously described [18].

\section{ELISA VEGF secretion}

SKOV-3 cells $\left(8 \times 10^{3}\right.$ per well) were seeded in 96-well plates and cultured overnight. The next day, medium was replaced with fresh RPMI 1640 or medium containing chA21 $(5 \mu \mathrm{g} / \mathrm{ml})$, trastuzumab $(5 \mu \mathrm{g} / \mathrm{ml})$, or chA21 plus trastuzumab $(5+5 \mu \mathrm{g} / \mathrm{ml})$ for $12 \mathrm{~h}$. After the supernatant was collected, the concentration of VEGF were measured using an ELISA kit for human VEGF (R\&D Systems, USA) according to the manufacturer's instructions. The amount of VEGF in the supernatant was extrapolated from the VEGF standard curve and expressed in $\mathrm{pg} / \mathrm{ml}$. The levels of VEGF that could be detected in this assay ranged from $30-1200 \mathrm{pg} / \mathrm{ml}$.

\section{HUVECs proliferation}

SKOV-3 cells $\left(4 \times 10^{3}\right.$ per well) were seeded in 96-well plates. The next day cells were treated with chA21 (5 $\mu \mathrm{g} / \mathrm{ml})$, trastuzumab $(5 \mu \mathrm{g} / \mathrm{ml})$, or chA21 plus trastuzu$\mathrm{mab}(5+5 \mu \mathrm{g} / \mathrm{ml})$ for $48 \mathrm{~h}$. The supernatant was collected and frozen at $-20^{\circ} \mathrm{C}$ for the HUVECs proliferation assay. HUVECs were seeded in 96-well plates at a density of $5 \times 10^{3}$ per $100 \mu \mathrm{l}$ and allowed to adhere overnight. Next, $100 \mu \mathrm{l}$ of SKOV-3 supernatant was added to each well and HUVECs were cultured for $72 \mathrm{~h}$. The 
number of HUVECs was measured by the MTS assay according to the manufactor's introduction (Promega, USA).

\section{HUVECs Migration assay}

The HUVECs migration was assessed by Transwell assay ( $8 \mu \mathrm{m}$, Millipore, USA) in a double chamber co-culture system. Briefly, SKOV-3 cells $\left(1.5 \times 10^{4}\right)$ were plated into 24-well plates (bottom chambers) and cultured with medium or medium supplemented with chA21 $(5 \mu \mathrm{g} /$ $\mathrm{ml})$, trastuzumab $(5 \mu \mathrm{g} / \mathrm{ml})$, or chA21 $(5 \mu \mathrm{g} / \mathrm{ml})$ plus trastuzumab $(5 \mu \mathrm{g} / \mathrm{ml})$ for $24 \mathrm{~h}$. HUVECs $\left(8 \times 10^{3}\right.$ per well) were seeded in Matrigel pre-coated Transwell chamber (top chamber), then the Transwell chambers were incubated into the 24-well plates. After co-cultured for $48 \mathrm{~h}$, the top surfaces of the Transwell chambers were wiped with cotton swab. The migrated cells were fixed and stained with hematoxylin. Migration cells adhering to the undersurface of the filter were counted using an optical microscope $(\times 400)$. Data was shown as the mean of the number of migrated HUVECs in five representative fields.

\section{Western blotting analysis}

SKOV-3 cells were grown in 6-well dishes and treated with chA21 $(5 \mu \mathrm{g} / \mathrm{ml})$, trastuzumab $(5 \mu \mathrm{g} / \mathrm{ml})$, or both agents together $(5+5 \mu \mathrm{g} / \mathrm{ml})$ for $12 \mathrm{~h}$. After the medium was removed, cells were washed twice with cold PBS and lysed in a $0.3 \mathrm{ml}$ of radioimmunoprecipitation assay (RIPA) lysis buffer (20 mM sodium phosphate, $\mathrm{pH}$ 7.4, $150 \mathrm{mM} \mathrm{NaCl}, 1 \%$ Triton X-100, 5 mM EDTA, 5 $\mathrm{mM}$ phenylmethylsulfonyl fluoride, $10 \mathrm{mg} / \mathrm{ml}$ aprotinin, $10 \mathrm{mg} / \mathrm{ml}$ leupeptin, $250 \mathrm{mg} / \mathrm{ml}$ sodium vanadate) on ice. After removal of cell debris by centrifugation, protein concentration was determined by Lowry assay (BioRad, USA). Cell lysates were subjected to $8 \%$ sodium dodecyl sulfate-polyacrylamide gel electrophoresis (SDSPAGE) and then electrotransferred into the nitrocellulose membrane. After blocking with $5 \%$ defatted milk, the membrane was incubated separately with antibody against VEGF (1:500, Neomarkers), AKT (1:1000, Cell signaling Technology) or phospho-AKT (pAKT) at Ser473 (1:1000, Cell signaling Technology) for $2 \mathrm{~h}$ at room temperature. Sequently, the membrane was probed with horseradish peroxidase (HRP)-conjugated secondary goat anti-mouse antibody (1:10,000, Sigma) for $2 \mathrm{~h}$ at room temperature. Immunoreactive bands were developed with chemiluminescence (ECL) reagents (Pierce). The band were scanned for densitometric analysis using Image 1.42 software (NIH, USA).

\section{Statistical analysis}

Data are shown as means \pm standard deviation (SD). Statistical analyses of the data were performed using one-way ANOVA test by SPSS 13.0. Value of $P<0.05$ was considered statistically significant.

\section{Results}

Enhanced tumor growth inhibition by combination of chA21 with trastuzumab

Initially, we evaluated whether the chA21 plus trastuzumab treatment leads to better tumor inhibition in SKOV-3 xenografts. Female BALB/c nude mice were subcutaneously inoculated with human ovarian cancer cells SKOV-3 $\left(5 \times 10^{6}\right)$ into the left flank of mice. Mice were randomized and injected twice weekly via i.v with either normal saline control, chA21 (30 mg/kg), trastuzumab $(20 \mathrm{mg} / \mathrm{kg})$, or chA21 plus trastuzumab $(30+20$ $\mathrm{mg} / \mathrm{kg}$ ) for 21 days. Either chA21 or trastuzumab alone treatment resulted in an effective suppression of tumor volume (Fig. 1A) and tumor weight (Fig. 1B) at day 21. The tumor inhibition ratios by chA21 or trastuzumab
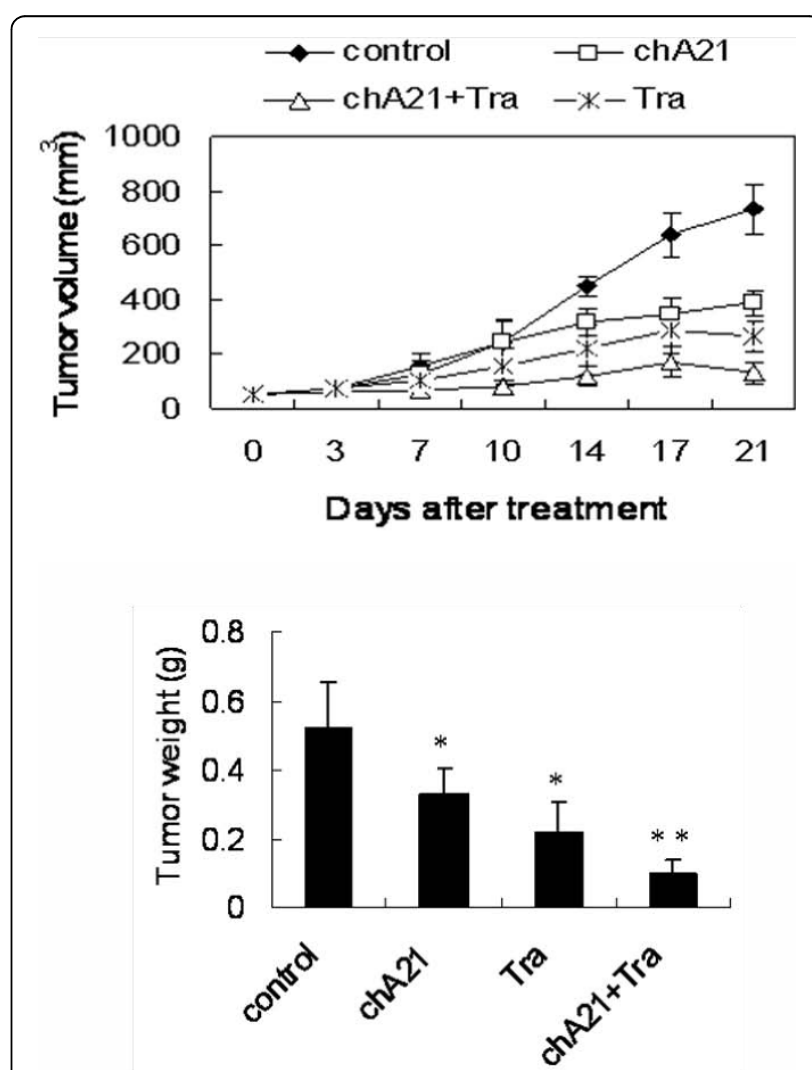

Figure 1 The tumor volume and the weight of SKOV-3 xenograft in the different treatment groups. (A) Either ChA21 or trastuzumab treatment cause a marked growth inhibition in SKOV-3 xenograft compared with the control $(P<0.01)$, and the combination of chA21 with trastuzumab treatment induced a more efficient efficacy than the each antibody alone $(P<0.05)$. (B) When the experiment ended, all tumors were removed and weighted. Results are representative of the mean \pm SD of 8 animals in each group. ${ }^{*}, P<0.01$ compared with control. ${ }^{*}, P<0.01$ compared with chA21 or trastuzumab alone. 
were $37 \%$ and $58 \%$, respectively $(P<0.01)$. Moreover, the combination of chA21 and trastuzumab resulted in an $81 \%$ inhibition in tumor weight compared with the control $(P<0.001)$, which is greater than single treatment $(P<0.01)$. In addition, complete tumor eradication was seen in one mice from the combination treatment group.

\section{Increased anti-angiogenesis efficacy by combination of chA21 with trastuzumab}

Angiogenesis plays an important role in cancer growth, we then examined whether the chA21 plus trastuzumab treatment leads to a more effective inhibition of angiogenesis than either treatment alone. MVD values were assessed by staining these with CD34 in tumor tissues that were removed from SKOV-3 xenografts. The most highly vascularized area of each tumor was identified on five high-powered fields were counted in this area of greatest vessel density. As shown in Fig. 2A and $2 \mathrm{~B}$, The number of MVD was $31 \%$ of the control in chA21 plus trastuzumab group, while this number was $56 \%$ in chA21 alone group and 54\% in trastuzumab alone group. So chA21 combined with trastuzumab treatment resulted in a marked inhibition of tumor MVD compared with the control $(P<0.001)$ and either of chA21 or trastuzumab alone treatment $(P<0.01)$.

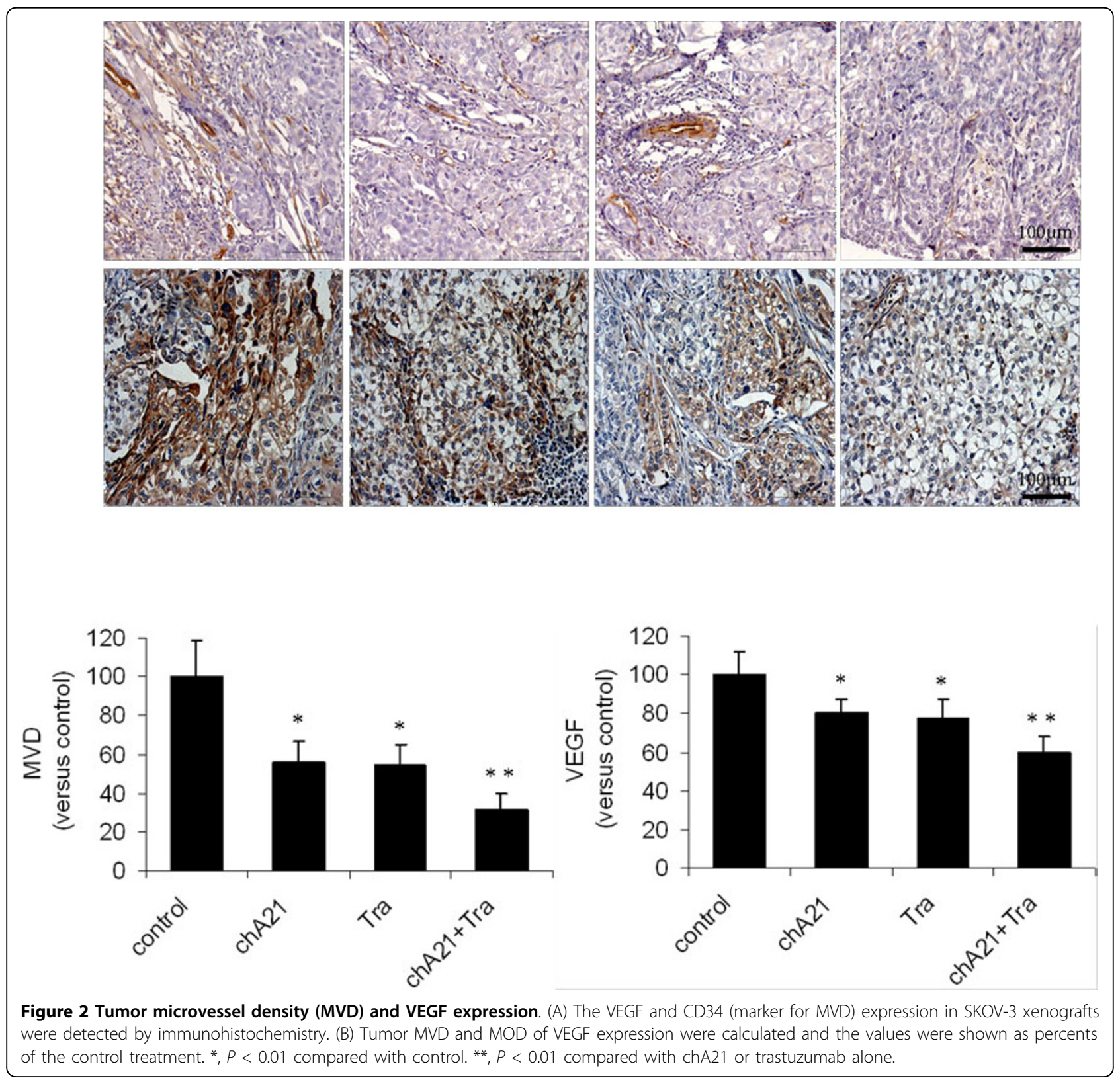


Similarly, the tumor mean optical density (MOD) values of VEGF in the chA21 plus trastuzumab treatment group were $60 \%$ of the control, lower than those of $80 \%$ and $77 \%$ in individual treatment groups of chA21 and trastuzumab, respectively $(P<0.01)$.

\section{Augmented down-regulation of VEGF expression induced by combination of chA21 with trastuzumab}

Previouly, we found HER2 antibody inhibits angiogenesis and downregulats VEGF expression. Hence, we explored the effect of antibody synergy on VEGF seceration by ELISA test. We examined the amount of VEGF secreted into the medium from SKOV-3 cells that were treated with chA21, trastuzumab, or chA21 plus trastuzumab for $12 \mathrm{~h}$. Compared with $1255.6 \pm 153.6 \mathrm{pg} / \mathrm{ml}$ in the control group, the level of secreted VEGF decreased $918.7 \pm 109.8 \mathrm{pg} / \mathrm{ml}$ in chA21 group $(P<$ $0.01), 839.1 \pm 137.8 \mathrm{pg} / \mathrm{ml}$ in trastuzumab group $(P<$ $0.01)$, and $583.5 \pm 87.7 \mathrm{pg} / \mathrm{ml}$ in the chA21 plus trastuzumab group $(P<0.001)$ (Fig. $3 \mathrm{~A})$.

We determined VEGF protein expression by Western blot upon HER2 antibody treatment for $12 \mathrm{~h}$ in SKOV-3

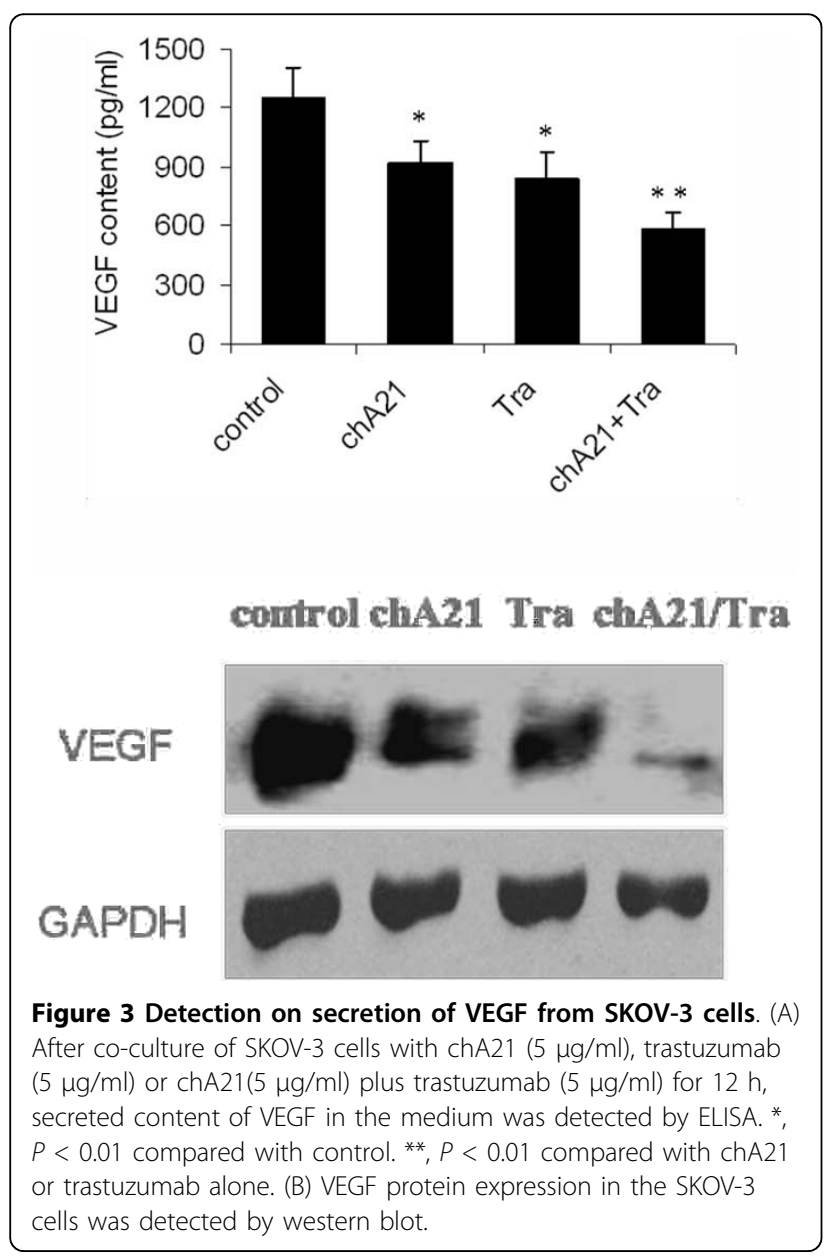

cells. In cosistent with ELISA data, we found treatment with chA21 or trastuzumab resulted in similar reduction of VEGF expression compared with the control. However, the combination of chA21 and trastuzumab induced a further inhibition of VEGF protein expression (Fig. 3B).

\section{Enhanced suppression of HUVECs proliferation and} migration by combination of chA21 with trastuzumab To further investigate the influence on the function of typical endothelial cells such as HUVECs, the effect of supernatant from SKOV-3 cells treated with antibodies on HUVECs proliferation was determined by the MTS assay. Compared with control group, HUVECs proliferation was inhibited by $33 \%$ in SKOV-3 supernatants treated with chA21 plus trastuzumab treatment group, 14\% in chA21 group $(P<0.05)$ and $16 \%$ trastuzumab group $(P<0.05)$ (Fig. 4A)

HUVECs were co-cultured with SKOV-3 supernatants for $48 \mathrm{~h}$. Migrated cells were stained and counted. As shown in Fig. $4 \mathrm{~B}$ and $4 \mathrm{C}$, the migrating capability of HUVECs was inhibited by SKOV-3 cell supernatant treated with chA21 plus trastuzumab compared with untreated cells $(P<0.001)$, chA21-treated cells $(P<$ $0.01)$ or trastuzumab-treated cells $(P<0.01)$. The number of migrated cells $(40 \times 10$ magnification) were $73 \%$, $70 \%$ or $48 \%$ in chA 21 , trastuzumab, or combined treatment group compared with the control (Fig. 4C). Our data demonstrated that chA21 or trastuzumab treatment suppressed HUVECs migration and combination of chA21 and trastuzumab induced a further suppression.

\section{Potent inhibition of pAKT activity by combination of chA21 with trastuzumab}

To detect the underlying molecular mechanism of angiogenesis, we investigated the effect of chA21 plus trastuzumab treatment on AKT activity, which is a crucial pathway in angiogenesis [19]. After SKOV-3 cells were treated with antibodies for $12 \mathrm{~h}$, the cell lysates were analyzed by Western blotting assay. As shown in Fig. 5A, pAKT (Ser ${ }^{473}$ ) expression were reduced by ChA21 or trastuzumab treatment, but more dramatic reduction was observed in the ChA21 plus trastuzumab treatment group. Total AKT protein were not altered significantly upon various interventions. As shown in Fig. 5B, The ratios of p-AKT/AKT were $0.61,0.65$ and 0.45 in chA21, trastuzumab and chA21 plus trastuzumab group. These data indicate that the ChA21 plus trastuzumab treatment cause greater inhibition of AKT expression compared with either treatment alone.

\section{Discussion}

It's well known that HER2-overexpressing tumors confer enhanced metastasis-related properties and resistance to 


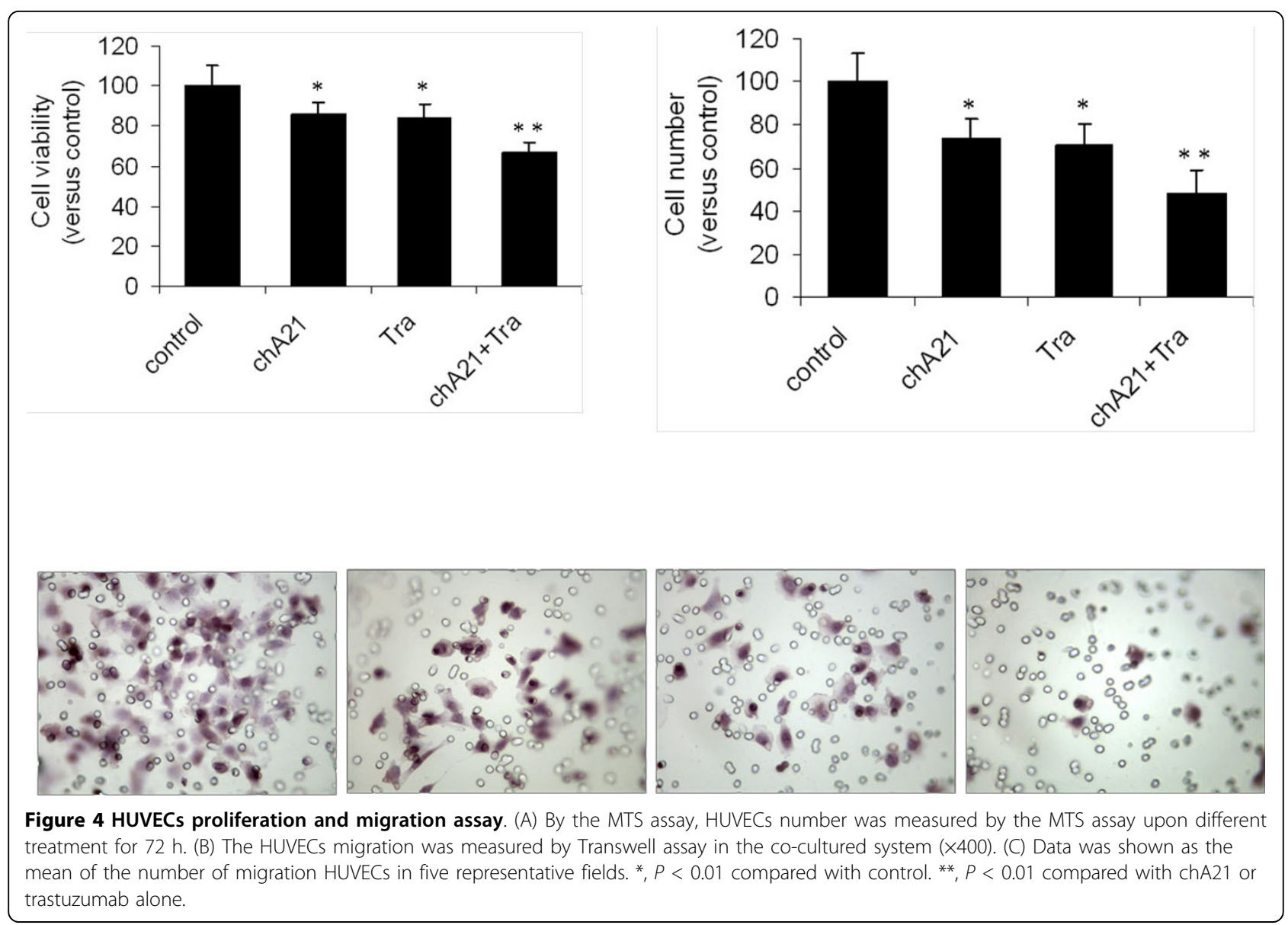

chemotherapeutic reagents which frequently result in poor clinical outcome. Trastuzumab as the first therapeutic anti-HER2 monoclonal antibody has been used in clinical treatment of HER2-overexpressing metastatic breast and gastric cancers $[20,21]$. It is also proposed to be a treatment option for patients with HER2-positive ovarian carcinoma [22]. However, poor responses and disease recurrences for trastuzumab therapy underscore for alternative treatments [23].

It is believed that angiogenesis is required for tumor growth and spread. HER2 signaling has been reported to be implicated in tumor angiogenesis [24,25]. Previously, we have developed a novel anti-HER2 chimeric antibody chA21 and this new antibody mainly binds to a distinct epitope on HER2 ECD I and inhibits tumor cells growth in vitro and in vivo $[10,16]$. In the present study we further explored the potential anti-angiogenic effects of chA21 on HER2-overexpressing ovarian carcinoma. Our data showed that chA21 and trastuzumab can inhibit tumor growth and angiogenesis in SKOV-3 xenograft model and the combination of trastuzumab with chA21 results in an enhanced effect.
Indeed, endothelial cells migration and proliferation is crucial for angiogenesis. Tumor cells induce angiogenesis by secreting various growth factors, such as VEGF, which binds its cognate receptor on endothelial cells and promotes these cells to proliferate and migrate $[26,27]$. Using ELISA kit to measuring the secreted VEGF from SKOV-3 cells, we found that chA21 could suppress VEGF expression and this synergism when combined chA21 with trastuzumab. Moreover, the synergism was confirmed by inhibition of HUVECs proliferation and migration when the endothelial cells were co-cultured with the supernatant from SKOV-3 cells treated with both trastuzumab and chA21 together. Therefore, the anti-angiogenesis capacity of the two antibodies alone and their synergy proved to be intrinsic.

Among signaling pathways induced by HER2 receptor, activation of the AKT kinase orchestrates a number of signaling pathways potentially involved in angiogenesis [19]. Our study revealed that chA21 could inhibit AKT expression with the capability similar to trastuzumab in SKOV-3 cell line. This result reflected the intrinsic 


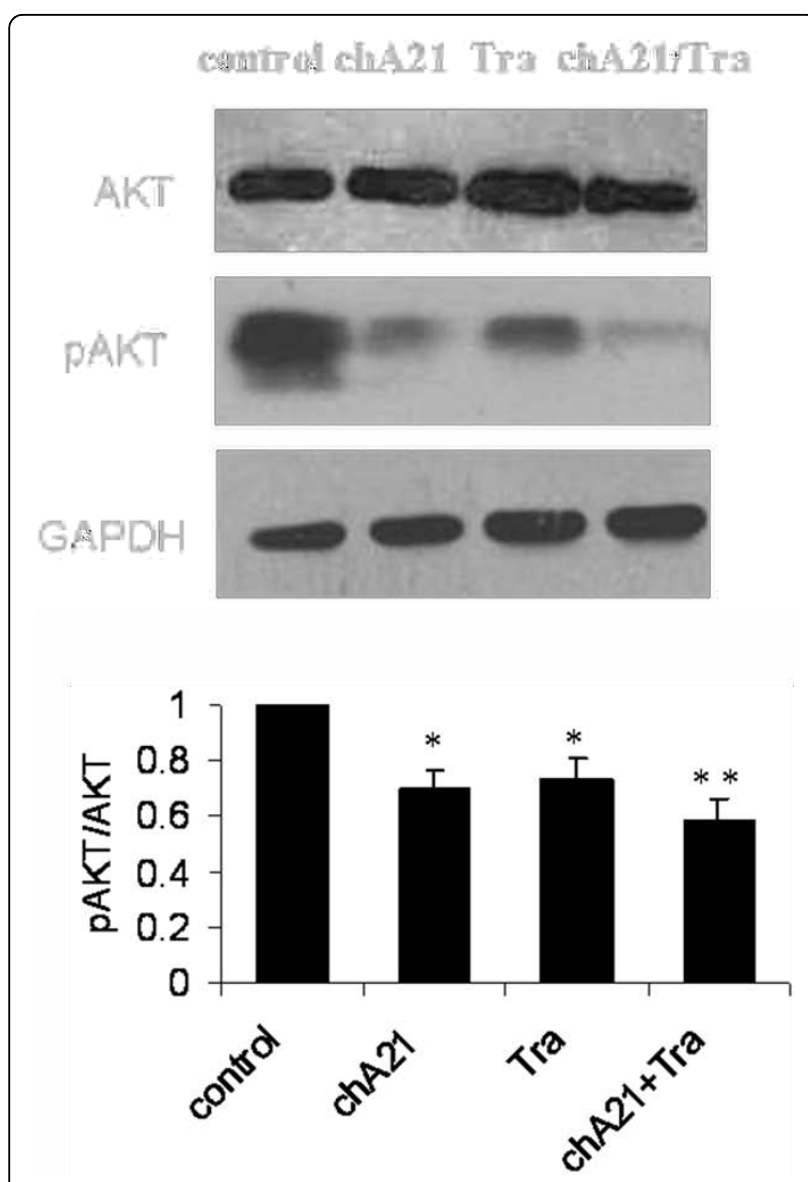

Figure 5 Protein expression of AKT and phopsho-AKT (pAKT) in xenograft tumors. (A) After SKOV-3 cells were treated with antibodies for $12 \mathrm{~h}$, the cell lysates were analyzed. PAKT $\left(\mathrm{Ser}^{473}\right)$ expression educed upon treament withchA21 or trastuzumab treatment and more dramatic reduction was observed with chA21 plus trastuzumab treatment. The levels of total AKT protein were not altered significantly upon various interventions. (B) The ratios of p-AKT/AKT were calculated and the values were shown as ratios compared with control, which was arbitrarily taken as 1.0. ${ }^{*}, P<$ 0.01 compared with control. ${ }^{*}, P<0.01$ compared with chA21 or trastuzumab alone.

properties of most tumor-inhibitory anti-HER2 antibodies to inhibit receptor-induced downstream signals at various efficiencies [28,29]. More importantly, the combination of chA21 with trastuzumab showed more significant potency on inhibiting the AKT. It may partly explain our findings that the combination of chA21 with trastuzumab could synergistically enhance the in vitro and in vivo anti-angiogenesis effects.

In conclusion, our study demonstrated the inhibition activities on tumor growth and angiogenesis of a novel anti-HER2 antibody chA21 alone and in combination with trastuzumab in vitro and in vivo. We found that the angiogenesis inhibition effect of chA21 could be enhanced by combination with trastuzumab, which might be mediated by synergism of chA21 and trastuzumab through inhibition of AKT expression. Therefore, chA21 may represent a unique anti-HER2 antibody with superior potentials as combination with other antiHER2 reagents for further therapy.

\section{Acknowledgements}

This work was granted by The Chinese Ministry of Science and Technology (Nos. 2006AA02A245 and 2009ZX09102-223), National Natural Science Foundation of China (No. 30873047) and Anhui Provincial Natural Science Foundation of China (No. 090413125). We would like to thank Hefei National Laboratory for Physical Sciences at Microscale, University of Science and Technology of China for technical help.

\section{Author details}

'Department of Pathology, Anhui Medical University, Meishan Road, Hefei, China. Institute of Clinical Pharmacology, Anhui Medical University, Meishan Road, Hefei, China. ${ }^{3}$ Affiliated Anhui Provincial Hospital, Anhui Medical University, Meishan Road, Hefei, China. ${ }^{4}$ Anhui Anke Biotechnology Co. Ltd, Haiguan Road, Hefei, China. ${ }^{5}$ School of Life Science, University of Science and Technology of China, Huangshan Road, Hefei, China.

\section{Authors' contributions}

AZ and GS designed and conducted the studies, carried out corresponding data analyses, and drafted the manuscript. TZ participated in the animal experiments. GZ, JL, LS, WW, BL, ZW and QW participated in study design, coordination and helped to draft the manuscript. All authors have read and approved this final manuscript.

\section{Competing interests}

The authors declare that they have no competing interests.

Received: 13 May 2010 Accepted: 19 August 2010

Published: 19 August 2010

\section{References}

1. Jemal A, Siegel R, Ward E, Hao Y, Xu J, Murray T, Thun MJ: Cancer statistics. CA Cancer J Clin 2008, 58(2):71-96.

2. Breedlove G, Busenhart C: Screening and detection of ovarian cancer. J Midwifery Womens Health 2005, 50(1):51-54.

3. Burstein HJ: The distinctive nature of HER2-positive breast cancers. N Engl J Med 2005, 353(16):1652-1654.

4. Slamon DJ, Godolphin W, Jones LA, Holt JA, Wong SG, Keith DE, Levin WJ, Stuart SG, Udove J, Ullrich A: Studies of the HER-2/neu proto-oncogene in human breast and ovarian cancer. Science 1989, 244(4905):707-712.

5. Verri E, Guglielmini P, Puntoni M, Perdelli L, Papadia A, Lorenzi P, Rubagotti A, Ragni N, Boccardo F: HER2/neu oncoprotein overexpression in epithelial ovarian cancer: evaluation of its prevalence and prognostic significance. Oncology 2005, 68(2-3):154-161.

6. Agus DB, Gordon MS, Taylor C, Natale RB, Karlan B, Mendelson DS, Press MF, Allison DE, Sliwkowski MX, Lieberman G: Phase I clinical study of pertuzumab, a novel HER dimerization inhibitor, in patients with advanced cancer. J Clin Oncol 2005, 23(11):2534-2543.

7. Wilken JA, Webster KT, Maihle NJ: Trastuzumab Sensitizes Ovarian Cancer Cells to EGFR-targeted Therapeutics. J Ovarian Res 2010, 3:7.

8. Cheng LS, Liu AP, Yang JH, Dong YQ, Li LW, Wang J, Wang CC, Liu J: Construction, expression and characterization of the engineered antibody against tumor surface antigen, p185(c-erbB-2). Cell Res 2003, 13(1):35-48.

9. Hu S, Li L, Qiao J, Guo Y, Cheng L, Liu J: Codon optimization, expression, and characterization of an internalizing anti-ErbB2 single-chain antibody in Pichia pastoris. Protein Expr Purif 2006, 47(1):249-257.

10. Hu S, Zhu Z, Li L, Chang L, Li W, Cheng L, Teng M, Liu J: Epitope mapping and structural analysis of an anti-ErbB2 antibody A21: Molecular basis for tumor inhibitory mechanism. Proteins 2008, 70(3):938-949.

11. Liu Y, Zhou H, Zhu J, Gao Y, Niu L, Liu J, Teng M: Crystallization and preliminary crystallographic studies of the single-chain variable fragment of antibody chA21 in complex with an $\mathrm{N}$-terminal fragment of 
ErbB2. Acta Crystallogr Sect F Struct Biol Cryst Commun 2009, 65(Pt 7):692-694.

12. Koukourakis MI, Manolas C, Minopoulos G, Giatromanolaki A, Sivridis E: Angiogenesis relates to estrogen receptor negativity, c-erbB-2 overexpression and early relapse in node-negative ductal carcinoma of the breast. Int I Surg Pathol 2003, 11(1):29-34.

13. Russell KS, Stern DF, Polverini PJ, Bender JR: Neuregulin activation of ErbB receptors in vascular endothelium leads to angiogenesis. Am J Physiol 1999, 277(6 Pt 2):H2205-2211.

14. Petit AM, Rak J, Hung MC, Rockwell P, Goldstein N, Fendly B, Kerbel RS: Neutralizing antibodies against epidermal growth factor and ErbB-2/neu receptor tyrosine kinases down-regulate vascular endothelial growth factor production by tumor cells in vitro and in vivo: angiogenic implications for signal transduction therapy of solid tumors. Am J Pathol 1997, 151(6):1523-1530.

15. Chung BH, Kim CK: Icariin stimulates angiogenesis by activating the MEK/ ERK-and PI3K/AKT/eNOS-dependent signal pathway in human endothelial cells. Biochem Biophys Res Commun 2008, 376:404-408.

16. Zhang AL, Xue H, Ling XG, Gao Y, Yang F, Cheng LS, Liu J, Wu Q: Anti-HER2 engineering antibody ChA21 inhibits growth and induces apoptosis of SKOV-3 cells. Journal of Experimental \& Clinical Cancer Research 2010, 29:23.

17. Liu S, Luo X, Li D, Zhang J, Qiu D, Liu W, She L, Yang Z: Tumor inhibition and improved immunity in mice treated with flavone from Cirsium japonicum DC. Int Immunopharmacol 2006, 6(9):1387-1393.

18. Vermeulen PB, Gasparini G, Fox SB, Toi M, Martin L, McCulloch P, Pezzella F, Viale G, Weidner N, Harris AL: Quantification of angiogenesis in solid human tumours: an international consensus on the methodology and criteria of evaluation. Eur J Cancer 1996, 32 A(14):2474-2484.

19. Dimmeler S, Zeiher AM: Akt takes center stage in angiogenesis signaling. Circulation Research 2000, 86(1):4-5.

20. Slamon DJ, Leyland-Jones B, Shak S, Fuchs H, Paton V, Bajamonde A, Fleming T, Eiermann W, Wolter J, Pegram M: Use of chemotherapy plus a monoclonal antibody against HER2 for metastatic breast cancer that overexpresses HER2. New England Journal of Medicine 2001, 344(11):783-792.

21. Roukos DH: Targeting gastric cancer with trastuzumab: new clinical practice and innovative developments to overcome resistance. Ann Surg Oncol 2010, 17(1):14-17.

22. McAlpine JN, Wiegand KC, Vang R, Ronnett BM, Adamiak A, Kobel M, Kalloger SE, Swenerton KD, Huntsman DG, Gilks CB: HER2 overexpression and amplification is present in a subset of ovarian mucinous carcinomas and can be targeted with trastuzumab therapy. Bmc Cancer 2009, 9:433.

23. Krop IE, Winer EP: Ten years of HER2-directed therapy: still questions after all these years. Breast Cancer Res Treat 2009, 113(2):207-209.

24. Blackwell KL, Dewhirst MW, Liotcheva V, Snyder S, Broadwater G, Bentley R, Lal A, Riggins G, Anderson S, Vredenburgh J: HER-2 gene amplification correlates with higher levels of angiogenesis and lower levels of hypoxia in primary breast tumors. Clin Cancer Res 2004, 10(12 Pt 1):4083-4088.

25. Schoppmann SF, Tamandl D, Roberts L, Jomrich G, Schoppmann A, Zwrtek R, Dubsky P, Gnant M, Jakesz R, Birner P: HER2/neu expression correlates with vascular endothelial growth factor- $C$ and lymphangiogenesis in lymph node-positive breast cancer. Ann Oncol 2009, 21(5):955-960.

26. Laakkonen P, Waltari M, Holopainen T, Takahashi T, Pytowski B, Steiner P, Hicklin D, Persaud K, Tonra JR, Witte L: Vascular endothelial growth factor receptor 3 is involved in tumor angiogenesis and growth. Cancer Res 2007, 67(2):593-599.

27. Lamszus K, Brockmann MA, Eckerich C, Bohlen P, May C, Mangold U, Fillbrandt $R$, Westphal M: Inhibition of glioblastoma angiogenesis and invasion by combined treatments directed against vascular endothelial growth factor receptor-2, epidermal growth factor receptor, and vascular endothelial-cadherin. Clin Cancer Res 2005, 11(13):4934-4940.

28. Ben-Kasus T, Schechter B, Lavi S, Yarden Y, Sela M: Persistent elimination of ErbB-2/HER2-overexpressing tumors using combinations of monoclonal antibodies: relevance of receptor endocytosis. Proc Natl Acad Sci USA 2009, 106(9):3294-3299.

29. Friedman LM, Rinon A, Schechter B, Lyass L, Lavi S, Bacus SS, Sela M, Yarden $Y$ : Synergistic down-regulation of receptor tyrosine kinases by combinations of mAbs: implications for cancer immunotherapy. Proc Natl Acad Sci USA 2005, 102(6):1915-1920. doi:10.1186/1757-2215-3-20

Cite this article as: Zhang et al:: Augmented inhibition of angiogenesis by combination of HER2 antibody chA21 and trastuzumab in human ovarian carcinoma xenograft. Journal of Ovarian Research 2010 3:20.

\section{Submit your next manuscript to BioMed Central and take full advantage of:}

- Convenient online submission

- Thorough peer review

- No space constraints or color figure charges

- Immediate publication on acceptance

- Inclusion in PubMed, CAS, Scopus and Google Scholar

- Research which is freely available for redistribution 